\title{
Effective collision strengths for transitions in Fe $\mathrm{XV}^{\star}$
}

\author{
K. M. Aggarwal ${ }^{1}$, F. P. Keenan ${ }^{1}$, and A. Z. Msezane $^{2}$ \\ 1 Department of Pure and Applied Physics, The Queen's University of Belfast, Belfast BT7 1NN, Northern Ireland, UK \\ 2 Center for Theoretical Studies of Physical Systems, Clark Atlanta University, Atlanta Ga 30304, USA
}

Received 10 June 2003 / Accepted 30 July 2003

\begin{abstract}
Collision strengths for transitions among the energetically lowest 53 fine-structure levels belonging to the $\left(1 s^{2} 2 s^{2} 2 p^{6}\right)$ $3 \ell^{2}, 3 \ell 3 \ell^{\prime}, 3 \mathrm{~s} 4 \ell$ and $3 \mathrm{p} 4 \mathrm{~s}$ configurations of $\mathrm{Fe} \mathrm{XV}$ are computed, over an electron energy range below 160 Ryd, using the Dirac Atomic $R$-matrix Code (DARC) of Norrington \& Grant (2003). Effective collision strengths, obtained after integrating the collision strengths over a Maxwellian distribution of electron energies, have also been calculated. These results of effective collision strengths are tabulated for all 1378 inelastic transitions over a wide temperature range of $10^{5}$ to $10^{7} \mathrm{~K}$. Comparisons are also made with other $R$-matrix calculations and the accuracy of the results is assessed.
\end{abstract}

Key words. atomic data - atomic processes

\section{Introduction}

Electron impact excitation of iron ions has been a subject of intense research over the last few years. This is primarily because iron is an abundant element, particularly in solar and fusion plasmas, and its lines are observed over almost all ionization stages. As a result of this, several scientists are busy in calculating atomic data for iron ions. Here, we present our calculations for excitation rate coefficients, in the form of effective collision strengths $(\Upsilon)$, for transitions in Mg-like Fe XV. The corresponding results for energy levels, radiative rates and collision strengths have already been reported in our earlier papers (Aggarwal et al. 1999, 2000, 2001; Deb et al. 1999).

Realising the importance of Fe XV, a number of workers in the past have performed a variety of calculations to compute atomic parameters, including energy levels, radiative rates (or oscillator strengths), collision strengths $(\Omega)$, and excitation rate coefficients - see Aggarwal et al. (1999) for references. Most of the earlier calculations have used the distorted wave (DW) method for the computations of $\Omega$, and have neglected the contribution of resonances while computing the values of $\Upsilon$. However, in the recent past Eissner et al. (1999) and Griffin et al. (1999) have reported their results of $\Upsilon$ for transitions among the lowest 35 and 45 levels, respectively. Both,

Send offprint requests to: K. M. Aggarwal,

e-mail: K.Aggarwal@qub.ac.uk

* Table 4 is only available in electronic form at the CDS via anonymous ftp to cdsarc.u-strasbg.fr $(130.79 .128 .5)$ or via http://cdsweb.u-strasbg.fr/cgi-bin/qcat?J/A+A/410/349
Eissner et al. and Griffin et al., employed the $R$-matrix program for the computations of $\Omega$, and have included configuration interaction (CI) and relativistic effects. Furthermore, a large range of partial waves has been included to ensure the convergence of $\Omega$, and resonances in a fine energy mesh are delineated to accurately determine the values of $\Upsilon$. However, there are some deficiencies in the calculations of Griffin et al., as has already been demonstrated in our earlier work (Aggarwal et al. 2000). The computed values of $\Omega$ of Eissner et al. are comparably accurate at energies above thresholds, but are underestimated by up to a factor of two in the threshold energy region. This is due to the inclusion of a limited range of partial waves in this region $(J \leq 9.5)$, which is not sufficient for the convergence of $\Omega$, especially for the allowed transitions. As a result of this, their results of $\Upsilon$ are underestimated by up to a factor of three for many transitions - see Aggarwal et al. (2001) for details and examples. Furthermore, the Eissner et al.'s results for $\Upsilon$ are confined to the lowest 35 levels belonging to the $\left(1 s^{2} 2 s^{2} 2 p^{6}\right) 3 s^{2}, 3 s 3 p, 3 p^{2}, 3 s 3 d, 3 p 3 d$ and $3 p^{2}$ configurations. However, many transitions involving the levels of the $3 \mathrm{~s} 4 \mathrm{~d}$ and $3 \mathrm{~s} 4 \mathrm{f}$ configurations have already been observed in solar plasmas (Acton et al. 1985). Therefore, apart from improving upon the available results of Eissner et al., there is a clear need to extend their calculations by including the higher lying levels of Fe XV. In our present calculations, we have included an additional 18 levels of the $3 \mathrm{~s} 4 \mathrm{~s}, 3 \mathrm{~s} 4 \mathrm{p}, 3 \mathrm{~s} 4 \mathrm{~d}, 3 \mathrm{~s} 4 \mathrm{f}$, and $3 \mathrm{p} 4 \mathrm{~s}$ configurations. This inclusion of additional levels not only extends the range of available transitions, but also improves upon those results due to the inclusion of resonances arising from the higher excited levels. Additionally, contrary to the Breit-Pauli 
$R$-matrix approach of Eissner et al., we are adopting the fully relativistic Dirac $R$-matrix approach for calculating $\Omega$, and report our results of effective collision strengths for all 1378 transitions among the lowest 53 levels of Fe XV.

\section{Atomic structure}

For the computation of wavefunctions, we have used the fully relativistic GRASP (General purpose Relativistic Atomic Structure Program) code of Dyall et al. (1989) with the option of extended average level (EAL), in which a weighted (proportional to $2 j+1$ ) trace of the Hamiltonian matrix is minimized. This produces a compromise set of orbitals describing closely lying states with moderate accuracy. Furthermore, we have included $\mathrm{CI}$ among the 14 configurations of Fe XV, which are: $\left(1 \mathrm{~s}^{2} 2 \mathrm{~s}^{2} 2 \mathrm{p}^{6}\right) 3 \mathrm{~s}^{2}, 3 \mathrm{~s} 3 \mathrm{p}, 3 \mathrm{p}^{2}, 3 \mathrm{~s} 3 \mathrm{~d}, 3 \mathrm{p} 3 \mathrm{~d}, 3 \mathrm{~d}^{2}, 3 \mathrm{~s} 4 \mathrm{~s}, 3 \mathrm{~s} 4 \mathrm{p}, 3 \mathrm{~s} 4 \mathrm{~d}$, $3 \mathrm{p} 4 \mathrm{~s}, 3 \mathrm{~s} 4 \mathrm{f}, 3 \mathrm{p} 4 \mathrm{p}, 3 \mathrm{p} 4 \mathrm{~d}$, and $3 \mathrm{p} 4 \mathrm{f}$. These configurations give rise to 87 fine-structure levels, which are listed in Table 1 of Deb et al. (1999).

The energies obtained for the 87 fine-structure levels of Fe XV have already been presented in our earlier paper (Deb et al. 1999). However, due to computational limitations, our calculations for $\Omega$ are restricted to the lowest 53 levels only, i.e. levels arising from the $3 \mathrm{p} 4 \mathrm{p}, 3 \mathrm{p} 4 \mathrm{~d}$, and $3 \mathrm{p} 4 \mathrm{f}$ configurations, have been excluded. In Table 1, we present our theoretical energies for these levels. Also included in this table are the experimentally compiled energies (Churilov et al. 1985 1989; Litzen \& Redfors 1987; Sugar \& Corliss 1985), and the theoretical results of Eissner et al. (1999) and Griffin et al. (1999), who have adopted the SuperStructure (SS) and Configuration Average Hartree-Fock (CAHF) programs of Eissner et al. (1974) and Froese-Fischer (1991), respectively. Both, Eissner et al. and Griffin et al., have also included CI, but the inclusion of relativistic effects is limited to one-body operators only. We note that experimental energies are not available for all the finestructure levels listed in Table 1. However, for those levels for which there are experimental energies, agreement with the present calculations is within $1.5 \%$, which is highly satisfactory. Moreover, the ordering of the energy levels is also the same between the theory and the experiment, except for two levels, namely 33 and 34, which differ with each other by only 0.006 Ryd. Similarly, the agreement between our present GRASP and earlier SS and CAHF level energies is better than $1 \%$, and the energy ordering differs only in one instance - see levels 32 and 33 of CAHF. Again, these levels are very close to each other, and differ by less than 0.003 Ryd.

The radiative rates obtained from GRASP using the above listed 14 configurations as well as those from using extensive CI (but only one-body relativistic operators) in the CIV3 program (Hibbert 1975), have already been presented and discussed in our earlier work (Deb et al. 1999). In that paper, extensive comparisons were also made between the length and velocity forms of the oscillator strengths (which are closely related to the radiative rates), as well as between the CIV3 and GRASP rates. To recapitulate, the agreement between the GRASP and CIV3 rates was better than $10 \%$ for a majority of the strong transitions $(f \geq 0.1)$, although the differences were higher for some of the transitions, especially those involving levels of the $3 \mathrm{p} 4 \mathrm{f}$ configuration (not included in the present calculations). For weaker transitions $(f<0.1)$, a difference of a factor of two between the GRASP and CIV3 rates was quite common, and was much larger for some of the transitions. The reason for the large differences for the weaker transitions was the inclusion of larger CI in the CIV3 calculations. However, in the fully relativistic present calculations for $\Omega$, it is not feasible to include so many configurations, and a selective inclusion of a few additional configurations does not improve the wavefunctions, as has been discussed in our previous work on Fe XXI (Aggarwal 1998). Therefore, we have preferred to use the GRASP wavefunctions for our present calculations of $\Omega$.

\section{Collision strengths}

For the computations of collision strengths $\Omega$, we have used the Dirac Atomic $R$-matrix Code (DARC) of Norrington \& Grant (2003). This program includes the relativistic effects in a systematic way, in both the target description and the scattering model. It is based on the $j j$ coupling scheme, and uses the Dirac-Coulomb Hamiltonian in the $R$-matrix approach. However, because of the inclusion of fine-structure in the definition of channel coupling, the matrix size of the Hamiltonian increases substantially, making the calculations computationally more expensive. The details of our calculations have already been reported in our earlier publication (Aggarwal et al. 1999). To summarize, the $R$-matrix radius has been adopted to be $4.44 \mathrm{au}$, and 25 continuum orbitals have been included for each channel angular momentum for the expansion of the wavefunction. This allows us to compute $\Omega$ up to an energy of 160 Ryd. In order to obtain convergence of $\Omega$ for all transitions and at all energies, we have included all partial waves with angular momentum $J \leq 39.5$, although a higher range would have been preferable for the convergence of allowed transitions, especially at higher energies. Unfortunately however, given the resources available to us, this was not possible. Nevertheless, to account for the inclusion of higher neglected partial waves, we have included a top-up, based on the sum rules of Burgess \& Tully (1978) for the allowed transitions, and a geometric series for the forbidden transitions.

A detailed comparison between our present (henceforth RM1) and those of Eissner et al. (1999: henceforth RM2) collision strengths for transitions among the lowest 10 finestructure levels of Fe XV has already been shown in our earlier work - see Table 2 and Figs. 1-6 of Aggarwal et al. (2000). For these transitions, there is a near total agreement between the two sets of $\Omega$ at all energies above thresholds. Here in Table 2 we compare the two sets of $\Omega$ for the $3 \mathrm{~s} 3 \mathrm{~d}-3 \mathrm{p} 3 \mathrm{~d}$ transitions, at three representative energies of 50,100 and 150 Ryd. Although there is excellent agreement (within 20\%) for many transitions, the differences between the two sets of $\Omega$ are rather large for a few transitions, such as 13-18 and 14-20, for which the discrepancy is up to $50 \%$. These transitions are allowed in the $j j$ or intermediate coupling scheme. Therefore, in Table 3 we show a comparison between the RM1 and RM2 oscillator strengths ( $f$ values in length form) for the $3 \mathrm{~s} 3 \mathrm{~d}-3 \mathrm{p} 3 \mathrm{~d}$ transitions. In general, the two sets of $\Omega$ are in accordance with the corresponding $f$ values, and the transitions for which the $f$-values are distinctly 
Table 1. Target levels of Fe XV and their threshold energies (in Ryd).

\begin{tabular}{|c|c|c|c|c|c|c|}
\hline Index & Configuration & Level & Expt. & GRASP & CAHF & $\mathrm{SS}$ \\
\hline 1 & $1 s^{2} 2 s^{2} 2 p^{6} 3 s^{2}$ & ${ }^{1} \mathrm{~S}_{0}$ & $0.0000^{a}$ & 0.0000 & 0.0000 & 0.000 \\
\hline 2 & $1 s^{2} 2 s^{2} 2 p^{6} 3 s 3 p$ & ${ }^{3} \mathrm{P}_{0}{ }^{0}$ & $2.1309^{a}$ & 2.1225 & 2.1160 & 2.126 \\
\hline 3 & $1 s^{2} 2 s^{2} 2 p^{6} 3 s 3 p$ & ${ }^{3} \mathrm{P}_{1}{ }^{0}$ & $2.1839^{a}$ & 2.1758 & 2.1667 & 2.177 \\
\hline 4 & $1 s^{2} 2 s^{2} 2 p^{6} 3 s 3 p$ & ${ }^{3} \mathrm{P}_{2}{ }^{0}$ & $2.3130^{a}$ & 2.3037 & 2.2887 & 2.298 \\
\hline 5 & $1 s^{2} 2 s^{2} 2 p^{6} 3 s 3 p$ & ${ }^{1} \mathrm{P}_{1}{ }^{0}$ & $3.2068^{a}$ & 3.2540 & 3.2383 & 3.245 \\
\hline 6 & $1 s^{2} 2 s^{2} 2 p^{6} 3 p^{2}$ & ${ }^{3} \mathrm{P}_{0}$ & $5.0532^{a}$ & 5.0756 & 5.0545 & 5.074 \\
\hline 7 & $1 s^{2} 2 s^{2} 2 p^{6} 3 p^{2}$ & ${ }^{1} \mathrm{D}_{2}$ & $5.0995^{a}$ & 5.1049 & 5.0853 & 5.105 \\
\hline 8 & $1 s^{2} 2 s^{2} 2 p^{6} 3 p^{2}$ & ${ }^{3} \mathrm{P}_{1}$ & $5.1450^{a}$ & 5.1652 & 5.1382 & 5.158 \\
\hline 9 & $1 s^{2} 2 s^{2} 2 p^{6} 3 p^{2}$ & ${ }^{3} \mathrm{P}_{2}$ & $5.3018^{a}$ & 5.3179 & 5.2823 & 5.303 \\
\hline 10 & $1 s^{2} 2 s^{2} 2 p^{6} 3 p^{2}$ & ${ }^{1} \mathrm{~S}_{0}$ & $6.0110^{a}$ & 6.0720 & 6.0408 & 6.056 \\
\hline 11 & $1 s^{2} 2 s^{2} 2 p^{6} 3 s 3 d$ & ${ }^{3} \mathrm{D}_{1}$ & $6.1854^{a}$ & 6.2112 & 6.2179 & 6.225 \\
\hline 12 & $1 s^{2} 2 s^{2} 2 p^{6} 3 s 3 d$ & ${ }^{3} \mathrm{D}_{2}$ & $6.1947^{a}$ & 6.2203 & 6.2290 & 6.236 \\
\hline 13 & $1 s^{2} 2 s^{2} 2 p^{6} 3 s 3 d$ & ${ }^{3} \mathrm{D}_{3}$ & $6.2095^{a}$ & 6.2344 & 6.2466 & 6.254 \\
\hline 14 & $1 s^{2} 2 s^{2} 2 p^{6} 3 s 3 d$ & ${ }^{1} \mathrm{D}_{2}$ & $6.9447^{a}$ & 7.0405 & 7.0573 & 7.062 \\
\hline 15 & $1 s^{2} 2 s^{2} 2 p^{6} 3 p 3 d$ & ${ }^{3} \mathrm{~F}_{2}{ }^{0}$ & $8.4588^{a}$ & 8.4754 & 8.4729 & 8.494 \\
\hline 16 & $1 s^{2} 2 s^{2} 2 p^{6} 3 p 3 d$ & ${ }^{3} \mathrm{~F}_{3}{ }^{0}$ & $8.5488^{a}$ & 8.5644 & 8.5596 & 8.581 \\
\hline 17 & $1 s^{2} 2 s^{2} 2 p^{6} 3 p 3 d$ & ${ }^{1} \mathrm{D}_{2}{ }^{0}$ & $8.6435^{a}$ & 8.6658 & 8.6529 & 8.674 \\
\hline 18 & $1 s^{2} 2 s^{2} 2 p^{6} 3 p 3 d$ & ${ }^{3} \mathrm{~F}_{4}{ }^{0}$ & $8.6539^{a}$ & 8.6679 & 8.6588 & 8.680 \\
\hline 19 & $1 s^{2} 2 s^{2} 2 p^{6} 3 p 3 d$ & ${ }^{3} \mathrm{D}_{1}{ }^{0}$ & $8.9566^{c}$ & 9.0127 & 8.9954 & 9.012 \\
\hline 20 & $1 s^{2} 2 s^{2} 2 p^{6} 3 p 3 d$ & ${ }^{3} \mathrm{P}_{2}{ }^{0}$ & $8.9624^{a}$ & 9.0148 & 9.0013 & 9.018 \\
\hline 21 & $1 s^{2} 2 s^{2} 2 p^{6} 3 p 3 d$ & ${ }^{3} \mathrm{D}_{3}{ }^{0}$ & $9.0658^{a}$ & 9.1205 & 9.1020 & 9.119 \\
\hline 22 & $1 s^{2} 2 s^{2} 2 p^{6} 3 p 3 d$ & ${ }^{3} \mathrm{P}_{0}{ }^{0}$ & $9.0752^{c}$ & 9.1276 & 9.1042 & 9.121 \\
\hline 23 & $1 s^{2} 2 s^{2} 2 p^{6} 3 p 3 d$ & ${ }^{3} \mathrm{P}_{1}{ }^{0}$ & $9.0784^{c}$ & 9.1314 & 9.1086 & 9.126 \\
\hline 24 & $1 s^{2} 2 s^{2} 2 p^{6} 3 p 3 d$ & ${ }^{3} \mathrm{D}_{2}{ }^{0}$ & $9.0819^{a}$ & 9.1352 & 9.1145 & 9.132 \\
\hline 25 & $1 s^{2} 2 s^{2} 2 p^{6} 3 p 3 d$ & ${ }^{1} \mathrm{~F}_{3}{ }^{0}$ & $9.6824^{a}$ & 9.8109 & 9.8215 & 9.830 \\
\hline 26 & $1 s^{2} 2 s^{2} 2 p^{6} 3 p 3 d$ & ${ }^{1} \mathrm{P}_{1}{ }^{0}$ & $9.7951^{c}$ & 9.9571 & 9.9347 & 9.944 \\
\hline 27 & $1 s^{2} 2 s^{2} 2 p^{6} 3 d^{2}$ & ${ }^{3} \mathrm{~F}_{2}$ & $12.4876^{b}$ & 12.5267 & 12.5361 & 12.552 \\
\hline 28 & $1 s^{2} 2 s^{2} 2 p^{6} 3 d^{2}$ & ${ }^{3} \mathrm{~F}_{3}$ & $12.5027^{b}$ & 12.5414 & 12.5527 & 12.570 \\
\hline 29 & $1 s^{2} 2 s^{2} 2 p^{6} 3 d^{2}$ & ${ }^{3} \mathrm{~F}_{4}$ & $12.5215^{b}$ & 12.5593 & 12.5748 & 12.592 \\
\hline 30 & $1 s^{2} 2 s^{2} 2 p^{6} 3 d^{2}$ & ${ }^{1} \mathrm{D}_{2}$ & $12.7814^{b}$ & 12.8494 & 12.8658 & 12.879 \\
\hline 31 & $1 s^{2} 2 s^{2} 2 p^{6} 3 d^{2}$ & ${ }^{3} \mathrm{P}_{0}$ & $12.8081^{b}$ & 12.8634 & 12.8680 & 12.882 \\
\hline 32 & $1 s^{2} 2 s^{2} 2 p^{6} 3 d^{2}$ & ${ }^{3} \mathrm{P}_{1}$ & $12.8140^{b}$ & 12.8689 & 12.9004 & 12.889 \\
\hline 33 & $1 s^{2} 2 s^{2} 2 p^{6} 3 d^{2}$ & ${ }^{3} \mathrm{P}_{2}$ & $12.8286^{b}$ & 12.8820 & 12.8754 & 12.914 \\
\hline 34 & $1 s^{2} 2 s^{2} 2 p^{6} 3 d^{2}$ & ${ }^{1} \mathrm{G}_{4}$ & $12.8225^{b}$ & 12.8968 & 12.9445 & 12.957 \\
\hline 35 & $1 s^{2} 2 s^{2} 2 p^{6} 3 d^{2}$ & ${ }^{1} \mathrm{~S}_{0}$ & $13.5510^{b}$ & 13.6816 & 13.7015 & 13.714 \\
\hline 36 & $1 s^{2} 2 s^{2} 2 p^{6} 3 s 4 s$ & ${ }^{3} S_{1}$ & $16.0718^{d}$ & 16.0518 & 16.1269 & $\ldots \ldots$ \\
\hline 37 & $1 s^{2} 2 s^{2} 2 p^{6} 3 s 4 s$ & ${ }^{1} \mathrm{~S}_{0}$ & ....... & 16.2776 & 16.4092 & ....... \\
\hline 38 & $1 s^{2} 2 s^{2} 2 p^{6} 3 s 4 p$ & ${ }^{3} \mathrm{P}_{0}{ }^{0}$ & ...... & 17.1312 & 17.2066 & ....... \\
\hline 39 & $1 s^{2} 2 s^{2} 2 p^{6} 3 s 4 p$ & ${ }^{3} \mathrm{P}_{1}^{0}$ & ....... & 17.1368 & 17.2206 & ....... \\
\hline 40 & $1 s^{2} 2 s^{2} 2 p^{6} 3 s 4 p$ & ${ }^{3} \mathrm{P}_{2}{ }^{0}$ & 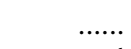 & 17.1994 & 17.2698 & ....... \\
\hline 41 & $1 s^{2} 2 s^{2} 2 p^{6} 3 s 4 p$ & ${ }^{1} \mathrm{P}_{1}{ }^{0}$ & $17.2227^{d}$ & 17.2069 & 17.3624 & ....... \\
\hline 42 & $1 s^{2} 2 s^{2} 2 p^{6} 3 s 4 d$ & ${ }^{3} \mathrm{D}_{1}$ & $18.5109^{d}$ & 18.5022 & 18.6002 & $\ldots \ldots$ \\
\hline 43 & $1 s^{2} 2 s^{2} 2 p^{6} 3 s 4 d$ & ${ }^{3} \mathrm{D}_{2}$ & $18.5171^{d}$ & 18.5093 & 18.6038 & $\ldots \ldots$ \\
\hline 44 & $1 s^{2} 2 s^{2} 2 p^{6} 3 s 4 d$ & ${ }^{3} \mathrm{D}_{3}$ & $18.5277^{d}$ & 18.5202 & 18.6192 & ....... \\
\hline 45 & $1 s^{2} 2 s^{2} 2 p^{6} 3 s 4 d$ & ${ }^{1} \mathrm{D}_{2}$ & $18.5472^{d}$ & 18.5404 & 18.6619 & $\ldots \ldots$ \\
\hline 46 & $1 s^{2} 2 s^{2} 2 p^{6} 3 p 4 s$ & ${ }^{3} \mathrm{P}_{0}{ }^{0}$ & $\ldots \ldots$ & 18.7170 & ....... & ....... \\
\hline 47 & $1 s^{2} 2 s^{2} 2 p^{6} 3 p 4 s$ & ${ }^{3} \mathrm{P}_{1}^{0}$ & ....... & 18.7533 & $\ldots \ldots$ & ....... \\
\hline 48 & $1 s^{2} 2 s^{2} 2 p^{6} 3 p 4 s$ & ${ }^{3} \mathrm{P}_{2}{ }^{0}$ & $\ldots \ldots$ & 18.9036 & $\ldots \ldots$ & $\ldots \ldots$ \\
\hline 49 & $1 s^{2} 2 s^{2} 2 p^{6} 3 p 4 s$ & ${ }^{1} \mathrm{P}_{1}{ }^{0}$ & ....... & 19.0532 & $\ldots \ldots$ & $\ldots \ldots$ \\
\hline 50 & $1 s^{2} 2 s^{2} 2 p^{6} 3 s 4 f$ & ${ }^{3} \mathrm{~F}_{2}{ }^{0}$ & $19.2145^{d}$ & 19.2033 & ...... & $\cdots$ \\
\hline 51 & $1 s^{2} 2 s^{2} 2 p^{6} 3 s 4 f$ & ${ }^{3} \mathrm{~F}_{3}{ }^{0}$ & $19.2153^{d}$ & 19.2052 & ....... & $\ldots .$. \\
\hline 52 & $1 s^{2} 2 s^{2} 2 p^{6} 3 s 4 f$ & ${ }^{3} \mathrm{~F}_{4}{ }^{0}$ & $19.2175^{d}$ & 19.2078 & $\ldots \ldots$ & $\ldots \ldots$ \\
\hline 53 & $1 s^{2} 2 s^{2} 2 p^{6} 3 s 4 f$ & ${ }^{1} \mathrm{~F}_{3}{ }^{0}$ & $19.3478^{d}$ & 19.3728 & $\ldots \ldots$ & ....... \\
\hline
\end{tabular}

${ }^{a}$ Churilov et al. (1985)

${ }^{b}$ Churilov et al. (1989).

${ }^{c}$ Litzen \& Redfors (1987).

${ }^{d}$ Sugar \& Corliss (1985). 
Table 2. Comparison of the present (RM1) collision strengths with those of Eissner et al. (RM2: 1999) for the 3s3d-3p3d transitions at three energies of 50, 100 and 150 Ryd. $\left(a \pm b \equiv a \times 10^{ \pm b}\right.$.)

\begin{tabular}{|c|c|c|c|c|c|c|c|}
\hline \multicolumn{2}{|c|}{ Transition } & \multicolumn{3}{|c|}{$\overline{\overline{\text { RM1 }}}$} & \multicolumn{3}{|c|}{$\overline{\mathrm{RM} 2}$} \\
\hline$i$ & J & 50.0 & 100.0 & 150.0 & 50.0 & 100.0 & 150.0 \\
\hline 11 & 15 & $3.1413-0$ & $3.7958-0$ & $4.4349-0$ & $3.3120-0$ & $3.8660-0$ & $4.2140-0$ \\
\hline 11 & 16 & $2.4478-3$ & $9.5303-4$ & $5.3452-4$ & $2.4400-3$ & $9.6070-4$ & $5.2950-4$ \\
\hline 11 & 17 & $3.7177-1$ & $4.4716-1$ & $5.1879-1$ & $4.1170-1$ & $4.8070-1$ & $5.2450-1$ \\
\hline 11 & 18 & $6.7714-5$ & $3.0076-5$ & $2.1444-5$ & $6.3190-5$ & $2.5820-5$ & $1.6380-5$ \\
\hline 11 & 19 & $5.8067-1$ & $6.9904-1$ & $8.0228-1$ & $6.2200-1$ & $7.3020-1$ & $7.9870-1$ \\
\hline 11 & 20 & $2.6911-1$ & $3.2405-1$ & $3.7231-1$ & $2.7950-1$ & $3.2750-1$ & $3.5820-1$ \\
\hline 11 & 21 & $9.4923-4$ & $4.0081-4$ & $2.3860-4$ & $8.9360-4$ & $3.8440-4$ & $2.2820-4$ \\
\hline 11 & 22 & $9.4654-1$ & $1.1409-0$ & $1.3060-0$ & $1.0060-0$ & $1.1850-0$ & $1.2980-0$ \\
\hline 11 & 23 & $2.0201-0$ & $2.4348-0$ & $2.7888-0$ & $2.1050-0$ & $2.4770-0$ & $2.7120-0$ \\
\hline 11 & 24 & $6.7922-1$ & $8.1753-1$ & $9.3697-1$ & $6.8330-1$ & $8.0160-1$ & $8.7760-1$ \\
\hline 11 & 25 & $2.0716-3$ & $7.5992-4$ & $3.9475-4$ & $1.8150-3$ & $6.8170-4$ & $3.5110-4$ \\
\hline 11 & 26 & $4.4311-3$ & $4.9597-3$ & $5.5138-3$ & $4.0620-3$ & $4.4600-3$ & $4.8170-3$ \\
\hline 12 & 15 & $8.4754-1$ & $1.0225-0$ & $1.1932-0$ & $8.8890-1$ & $1.0360-0$ & $1.1280-0$ \\
\hline 12 & 16 & $5.1011-0$ & $6.1616-0$ & $7.1821-0$ & $5.4020-0$ & $6.3100-0$ & $6.8840-0$ \\
\hline 12 & 17 & $9.1408-2$ & $1.0843-1$ & $1.2528-1$ & $8.9600-2$ & $1.0330-1$ & $1.1230-1$ \\
\hline 12 & 18 & $3.1962-3$ & $1.2305-3$ & $6.8507-4$ & $3.1210-3$ & $1.2140-3$ & $6.6300-4$ \\
\hline 12 & 19 & $2.0363-0$ & $2.4548-0$ & $2.8165-0$ & $2.1350-0$ & $2.5090-0$ & $2.7450-0$ \\
\hline 12 & 20 & $2.0357-1$ & $2.4378-1$ & $2.7946-1$ & $2.3940-1$ & $2.7970-1$ & $3.0570-1$ \\
\hline 12 & 21 & $1.0968-0$ & $1.3218-0$ & $1.5167-0$ & $1.1000-0$ & $1.2910-0$ & $1.4140-0$ \\
\hline 12 & 22 & $9.0858-4$ & $3.3872-4$ & $1.7973-4$ & $9.0400-4$ & $3.4200-4$ & $1.8000-4$ \\
\hline 12 & 23 & $7.3615-1$ & $8.8585-1$ & $1.0134-0$ & $8.1700-1$ & $9.6140-1$ & $1.0520-0$ \\
\hline 12 & 24 & $3.2166-0$ & $3.8781-0$ & $4.4434-0$ & $3.3510-0$ & $3.9420-0$ & $4.3150-0$ \\
\hline 12 & 25 & $1.2988-2$ & $1.2868-2$ & $1.3857-2$ & $1.2770-2$ & $1.2710-2$ & $1.3320-2$ \\
\hline 12 & 26 & $1.6686-2$ & $1.9371-2$ & $2.1709-2$ & $1.5770-2$ & $1.8010-2$ & $1.9640-2$ \\
\hline 13 & 15 & $1.3742-2$ & $1.6523-2$ & $1.9250-2$ & $1.4070-2$ & $1.6360-2$ & $1.7790-2$ \\
\hline 13 & 16 & $1.1479-0$ & $1.3837-0$ & $1.6112-0$ & $1.1800-0$ & $1.3780-0$ & $1.5020-0$ \\
\hline 13 & 17 & $2.2731-1$ & $2.7121-1$ & $3.1358-1$ & $2.1180-1$ & $2.4560-1$ & $2.6720-1$ \\
\hline 13 & 18 & $7.9866-0$ & $9.6400-0$ & $1.1211+1$ & $7.7690-0$ & $7.6310-0$ & $7.2780-0$ \\
\hline 13 & 19 & $1.0601-3$ & $4.3084-4$ & $2.5188-4$ & $1.0470-3$ & $4.4140-4$ & $2.6220-4$ \\
\hline 13 & 20 & $3.9626-0$ & $4.7762-0$ & $5.4826-0$ & $4.1950-0$ & $4.9360-0$ & $5.3970-0$ \\
\hline 13 & 21 & $4.7953-0$ & $5.7808-0$ & $6.6291-0$ & $5.0160-0$ & $5.9020-0$ & $6.4600-0$ \\
\hline 13 & 22 & $1.0506-4$ & $6.5478-5$ & $5.5224-5$ & $1.0820-4$ & $7.4220-5$ & $6.5360-5$ \\
\hline 13 & 23 & $7.7801-4$ & $3.6065-4$ & $2.3878-4$ & $7.8330-4$ & $3.7170-4$ & $2.4790-4$ \\
\hline 13 & 24 & $5.3263-1$ & $6.3970-1$ & 7.3184-1 & $6.3170-1$ & $7.4180-1$ & $8.1150-1$ \\
\hline 13 & 25 & $4.3548-2$ & $4.8783-2$ & $5.4325-2$ & $3.9400-2$ & $4.3430-2$ & $4.6850-2$ \\
\hline 13 & 26 & $1.6531-3$ & $5.8696-4$ & $3.0190-4$ & $1.5420-3$ & $5.5760-4$ & $2.8380-4$ \\
\hline 14 & 15 & $2.7442-1$ & $3.4563-1$ & $3.5495-1$ & 2.9910-1 & $3.4630-1$ & $3.7480-1$ \\
\hline 14 & 16 & $5.3826-2$ & $6.6310-2$ & $6.7431-2$ & $5.0300-2$ & $5.7390-2$ & $6.2010-2$ \\
\hline 14 & 17 & $1.9954-0$ & $2.4810-0$ & $2.6837-0$ & $2.1410-0$ & $2.4910-0$ & $2.7010-0$ \\
\hline 14 & 18 & $1.9971-3$ & $6.8223-4$ & $3.5259-4$ & $1.9430-3$ & $6.7330-4$ & $3.4240-4$ \\
\hline 14 & 19 & $3.5906-2$ & $4.1900-2$ & $4.7048-2$ & $3.6350-2$ & $4.0730-2$ & $4.3850-2$ \\
\hline 14 & 20 & $6.1278-2$ & $7.1889-2$ & $8.0182-2$ & $5.3790-2$ & $6.0160-2$ & $6.4760-2$ \\
\hline 14 & 21 & $3.6946-2$ & $3.9504-2$ & $4.5036-2$ & $3.6670-2$ & $3.8090-2$ & $4.0240-2$ \\
\hline 14 & 22 & $5.1061-4$ & $1.7603-4$ & $8.8350-5$ & $4.7770-4$ & $1.6820-4$ & $8.3350-5$ \\
\hline 14 & 23 & $2.3381-3$ & $1.1894-3$ & $9.5158-4$ & $2.0650-3$ & $9.7900-4$ & $6.9320-4$ \\
\hline 14 & 24 & $8.2149-3$ & $6.6399-3$ & $6.8872-3$ & $9.9650-3$ & $8.7470-3$ & $8.7670-3$ \\
\hline 14 & 25 & $1.2581+1$ & $1.5253+1$ & $1.7579+1$ & $1.3610+1$ & $1.6050+1$ & $1.7580+1$ \\
\hline 14 & 26 & $3.3882-0$ & $4.0996-0$ & $4.6964-0$ & $3.7030-0$ & $4.3770-0$ & $4.7980-0$ \\
\hline
\end{tabular}

different, such as 12-20, 13-24, 14-23 and 14-24, so are the values of $\Omega$. However, the $13-18\left(3 \mathrm{~s} 3 \mathrm{~d}{ }^{3} \mathrm{D}_{3}-3 \mathrm{p} 3 \mathrm{~d}{ }^{3} \mathrm{~F}_{4}^{\mathrm{o}}\right)$ transition is particularly noteworthy, because its $f$-value is comparatively high and both calculations report comparable results ( 0.15 and 0.16 in GRASP/RM1 and SS/RM2, respectively), but the $\Omega$ values are strikingly different (by up to $50 \%$ ). 
Table 3. Comparison between our GRASP (Deb et al. 1999) and Eissner et al.'s SuperStructure (SS: 1999) oscillator strengths (in length form) for the $3 \mathrm{~s} 3 \mathrm{~d}-3 \mathrm{p} 3 \mathrm{~d}$ transitions in Fe XV. $\left(a \pm b \equiv a \times 10^{ \pm b}\right.$.)

\begin{tabular}{rrrrrrrr}
\hline \multicolumn{2}{c}{ Transition } & \multicolumn{2}{c}{$f_{L}$} & \multicolumn{3}{c}{ Transition } & \multicolumn{2}{c}{$f_{L}$} \\
$i$ & $j$ & GRASP & SS & $i$ & $j$ & GRASP & SS \\
\hline 11 & 15 & $0.1261-0$ & $0.1362-0$ & 13 & 15 & $0.2365-3$ & $0.2393-3$ \\
11 & 17 & $0.1646-1$ & $0.1886-1$ & 13 & 16 & $0.2031-1$ & $0.2146-1$ \\
11 & 19 & $0.3102-1$ & $0.3350-1$ & 13 & 17 & $0.4018-2$ & $0.4009-2$ \\
11 & 20 & $0.1403-1$ & $0.1513-1$ & 13 & 18 & $0.1513-0$ & $0.1623-0$ \\
11 & 22 & $0.5439-1$ & $0.5773-1$ & 13 & 20 & $0.9184-1$ & $0.9674-1$ \\
11 & 23 & $0.1165-0$ & $0.1214-0$ & 13 & 21 & $0.1163-0$ & $0.1216-0$ \\
11 & 24 & $0.3933-1$ & $0.3960-1$ & 13 & 24 & $0.1249-1$ & $0.1511-1$ \\
11 & 26 & $0.3105-3$ & $0.2952-3$ & 13 & 25 & $0.1218-2$ & $0.1164-2$ \\
12 & 15 & $0.2012-1$ & $0.2168-1$ & 14 & 15 & $0.3466-2$ & $0.4036-2$ \\
12 & 16 & $0.1288-0$ & $0.1397-0$ & 14 & 16 & $0.7027-3$ & $0.7226-3$ \\
12 & 17 & $0.2402-2$ & $0.2380-2$ & 14 & 17 & $0.3029-1$ & $0.3376-1$ \\
12 & 19 & $0.6623-1$ & $0.6922-1$ & 14 & 19 & $0.6373-3$ & $0.6948-3$ \\
12 & 20 & $0.6189-2$ & $0.7592-2$ & 14 & 20 & $0.1086-2$ & $0.1024-2$ \\
12 & 21 & $0.3710-1$ & $0.3772-1$ & 14 & 21 & $0.6179-3$ & $0.6760-3$ \\
12 & 23 & $0.2502-1$ & $0.2776-1$ & 14 & 23 & $0.9642-5$ & $0.6662-5$ \\
12 & 24 & $0.1111-0$ & $0.1157-0$ & 14 & 24 & $0.7323-4$ & $0.1410-3$ \\
12 & 25 & $0.4067-3$ & $0.4586-3$ & 14 & 25 & $0.4069-0$ & $0.4440-0$ \\
12 & 26 & $0.7401-3$ & $0.7210-3$ & 14 & 26 & $0.1177-0$ & $0.1265-0$ \\
\hline
\end{tabular}

Since this is a strong allowed transition, the $\Omega$ values should increase with increasing energy as is the case in our calculations, and for a majority of other transitions in both calculations, but the RM2 values of $\Omega$ are unexpectedly decreasing. There may be a few more anomalies like this in any of the two calculations, but in general there is a broad agreement between the two sets of $\Omega$, which is highly satisfactory. The differences for a few transitions between the two sets of $\Omega$ are only due to the choice of wavefunctions, and hence CI, as our calculations include 14 configurations whereas those of RM2 only 6 .

\section{Effective collision strengths}

Effective collision strengths $\Upsilon$ are obtained after integrating $\Omega$ over a Maxwellian distribution of electron velocities, i.e.

$\Upsilon\left(T_{\mathrm{e}}\right)=\int_{0}^{\infty} \Omega(E) \exp \left(-E_{j} / k T_{\mathrm{e}}\right) \mathrm{d}\left(E_{j} / k T_{\mathrm{e}}\right)$,

where $E_{j}$ is the incident energy of the electron with respect to the final state of the transition, $k$ is Boltzmann's constant, and $T_{\mathrm{e}}$ is the electron temperature in K. Once the value of $\Upsilon$ is known for a transition, the corresponding value of the excitation $q(i, j)$ and de-excitation $q(j, i)$ rate coefficients can be easily obtained from the following simple relations:

$q(i, j)=\frac{8.63 \times 10^{-6}}{\omega_{i} T_{\mathrm{e}}^{1 / 2}} \Upsilon \exp \left(-E_{i j} / k T_{\mathrm{e}}\right) \quad \mathrm{cm}^{3} \mathrm{~s}^{-1}$

and

$q(j, i)=\frac{8.63 \times 10^{-6}}{\omega_{j} T_{\mathrm{e}}^{1 / 2}} \Upsilon \quad \mathrm{cm}^{3} \mathrm{~s}^{-1}$,

where $\omega_{i}$ and $\omega_{j}$ are the statistical weights of the initial $(i)$ and final $(j)$ states, respectively, and $E_{i j}$ is the transition energy.
Since the threshold energy region is dominated by numerous resonances, $\Omega$ must be computed at a large number of energies in order to delineate these resonances. We have performed our calculations of $\Omega$ at over 9380 energies in the threshold region. Close to thresholds $(\sim 0.1$ Ryd above a threshold) the energy mesh is $0.001 \mathrm{Ryd}$, and away from thresholds is 0.002 Ryd. This energy mesh is comparable to the uniform 0.002 Ryd energy mesh of RM2. Thus care has been taken to include as many resonances as possible, and in as fine a resolution as computationally feasible. The values of $\Upsilon$ so computed are listed in Table 4 at a series of electron temperatures in the range $10^{5}$ to $10^{7} \mathrm{~K}$, which is sufficient for the application of data in solar, astrophysical, fusion and laser plasmas.

The $\Upsilon$ values for transitions among the lowest 10 finestructure levels, i.e. $\left(3 \mathrm{~s}^{2}\right){ }^{1} \mathrm{~S}_{0}$, $(3 \mathrm{~s} 3 \mathrm{p}){ }^{1} \mathrm{P}_{1}^{0},{ }^{3} \mathrm{P}_{0,1,2}^{0}$, and $\left(3 \mathrm{p}^{2}\right)$ ${ }^{1} \mathrm{~S}_{0},{ }^{1} \mathrm{D}_{2}$ and ${ }^{3} \mathrm{P}_{0,1,2}$, have already been compared with those of RM2 in our earlier paper (Aggarwal et al. 2001). The $\Upsilon$ values of RM2 were found to be lower by up to a factor of three for some of the transitions, especially the allowed ones. This was primarily due to the non-convergence of their values of $\Omega$ at energies below 9.8 Ryd - see Aggarwal et al. for details. Generally their results for transitions with upper levels $n \leq 26$ $\left(3 \mathrm{p} 3 \mathrm{~d}^{1} \mathrm{P}_{1}^{0}\right)$ are underestimated. Their results for other transitions may also be underestimated, because their calculations are confined to the lowest 35 fine-structure levels of Table 1. As a result of this, resonances arising from the higher lying levels have not been taken into account. Similarly, our results will be comparatively less accurate for transitions with upper levels $n \geq 45\left(3 \mathrm{~s} 4 \mathrm{~d}^{1} \mathrm{D}_{2}\right)$, because we too have neglected levels arising from the $3 \mathrm{p} 4 \mathrm{p}, 3 \mathrm{p} 4 \mathrm{~d}$ and $3 \mathrm{p} 4 \mathrm{f}$ configurations.

It may also be noted that the $\Upsilon$-values listed here in Table 4 are different for a few transitions (such as: 1-6, 18, 3-7, 5-6 and 5-7) from those reported in our earlier publication (Aggarwal et al. 2001). This is mainly because three 
improvements have been made over those results. Firstly, the values of $\Omega$ included in that work at an energy of 20 Ryd were mistakenly adopted from our earlier (inconclusive and preliminary) results, which affected the interpolated $\Omega$ values in the energy range of $\sim 19.5-25.0$ Ryd. As a result of this, our values of $\Upsilon$ were affected particularly at the lower temperatures. Secondly, our values of $\Omega$ for some of the allowed transitions were underestimated, particularly towards the higher end of our energy range, see for example, Fig. 5 of Aggarwal et al. (2000) for the $3 \mathrm{~s} 3 \mathrm{p}^{1} \mathrm{P}_{1}^{0}-3 \mathrm{p}^{2}{ }^{3} \mathrm{P}_{0}(5-6)$ transition. This was due to an error in our top-up procedure, which has now been corrected. As a result of this, our results of $\Upsilon$ have been affected for some of the allowed transitions (such as: 1-3 and 5-9), particularly towards the higher end of the temperature range. Finally, in our earlier work (Aggarwal et al. 2001), those spurious resonances which were higher than a factor of 1000 , compared to the background $\Omega$, were deleted. In the present work we have adopted this factor to be 10000 , which has been found to be more appropriate after further experimentation with the calculations, experience on other ions such as Fe XXI, and discussions with our colleagues. This has affected the results of $\Upsilon$ for a few transitions, particularly towards the lower end of the temperature range. However, due to this adopted factor of 10000 the uncertainty of our $\Upsilon$ results for some of the transitions is higher at lower temperatures than the higher ones.

In Table 5 we compare the RM1 and RM2 results of $\Upsilon$ for the $3 \mathrm{~s} 3 \mathrm{~d}-3 \mathrm{p} 3 \mathrm{~d}$ transitions at three common temperatures of $10^{5}, 10^{6}$ and $10^{7} \mathrm{~K}$. For many transitions there is a fair amount of agreement ( $20 \%)$ among the two calculations, but for some transitions the differences are larger, and as expected and explained above, our results are generally higher. For example, our values of $\Upsilon$ for the ${ }^{14-18}\left({ }^{1} \mathrm{D}_{2}-{ }^{3} \mathrm{~F}_{4}^{\mathrm{o}}\right)$ transition are higher by a factor of three. Since this is a forbidden transition, we cannot compare the $f$-values to identify the differences. However, in Fig. 1a we show the variation of $\Omega$ over a small energy range below 12.5 Ryd. The collision strengths of RM2 for this transition are shown in Fig. 1b. A close examination of these two figures immediately reveals why our values of $\Upsilon$ are considerably higher. This is mainly for two reasons. Firstly, the background $\Omega$ of RM2 are lower by about a factor of three at energies below 9.8 Ryd. This is due to the non-convergence of their $\Omega$ in this energy range, as discussed above and demonstrated earlier (Aggarwal et al. 2001). Secondly, in our calculations we observe comparatively more resonances, because our calculations are in the $j j$ coupling scheme and have been performed in a finer energy mesh. However, the major elevation of our $\Upsilon$ values is from the former factor. Although 14-18 is a forbidden transition $\left({ }^{1} \mathrm{D}_{2}-{ }^{3} \mathrm{~F}_{4}^{\mathrm{o}}\right)$, partial waves with $J>9.5$ have made a significant contribution. A very good agreement (apart from a few narrow resonances) between the RM1 and RM2 values of $\Omega$ at energies above 9.8 Ryd further proves that the RM2 results are fully converged, but not at the lower energies, and this has resulted in the underestimation of their $\Upsilon$ values.

Finally, in Table 6 we compare the RM1 and RM2 data of $\Upsilon$ for the $3 \mathrm{~s} 3 \mathrm{~d}-3 \mathrm{~d}^{2}$ transitions at three temperatures of $10^{5}$, $10^{6}$ and $10^{7} \mathrm{~K}$. These transitions (generally) do not suffer from the problem of non-convergence of $\Omega$, and the differences in $\Upsilon$ values are primarily either due to the resonances from the higher lying levels or the differences in the $\Omega$ values themselves. Generally the two sets of data agree within $\sim 10 \%$, but for about $25 \%$ of these transitions the differences are up to a factor of two - see, for example, 12-30, 13-30 and 14-33 transitions. For all of these transitions our values of $\Omega$ are lower by a factor of two, and hence our $\Upsilon$ are also correspondingly lower. Our lower values of $\Omega$ are due to our choice of (better) wavefunctions, as discussed earlier.

\section{Conclusions}

In this paper we have reported the values of effective collision strengths for transitions among the lowest 53 fine-structure levels of Fe XV. Relativistic effects and CI have been included while generating the wavefunctions, and a large range of partial waves has been included to ensure the convergence of $\Omega$. Additionally, resonances have been delineated in a fine energy mesh while computing $\Upsilon$. Thus care has been taken to obtain the results as accurately as possible and computationally feasible.

A comparison has also been made with the earlier available data of Eissner et al. (1999). For collision strengths, there is an excellent agreement for many transitions but only at energies above thresholds. At energies below $9.8 \mathrm{Ryd}$, the $\Omega$ values of Eissner et al. are not converged due to the inclusion of limited number of partial waves. On the other hand, the differences for some of the transitions at higher energies are often a factor of two. This is due to the different choices of wavefunctions adopted in the calculations. Both calculations include CI among the internal orbitals only. Additionally, the RM1 and RM2 calculations have included CI among 14 and 6 configurations, respectively. Since our calculations include comparatively larger CI as well as all relativistic operators (the RM2 calculations include only one-body relativistic operators), the generated wavefunctions are expected to be comparatively more accurate. The differences with the RM2 calculations for the $\Upsilon$ values are up to a factor of three for some transitions, and our results are generally higher. This is mainly because their values of $\Omega$ are not converged at lower energies and resonances arising from higher lying levels have been neglected.

We do not see any apparent deficiency in our calculations but refrain from assessing the accuracy of our results, because of past experience. Due to the presence (or absence) of a large number of resonances in the thresholds region, especially those closer to thresholds, the reported values of $\Upsilon$ could easily show variations of $\sim 20 \%$, particularly at the lower end of the temperature range. The $\Upsilon$ values at the higher end of the temperature range are likely to be comparatively more accurate. However, since our calculations are restricted to the lowest 53 levels of Fe XV, resonances arising from the higher lying levels are not taken into account. This will affect the values of $\Upsilon$, particularly for those transitions which have upper levels $n \geq 45$ (i.e. $3 \mathrm{~s} 4 \mathrm{~d}^{1} \mathrm{D}_{2}$ - see Table 1). Similarly, there can also be some additional uncertainty in our $\Upsilon$ values towards the higher temperature range of our calculations. This is because our calculations of $\Omega$ are restricted to energies below $160 \mathrm{Ryd}$, whereas a higher range of energy is desirable in order to improve the accuracy of 

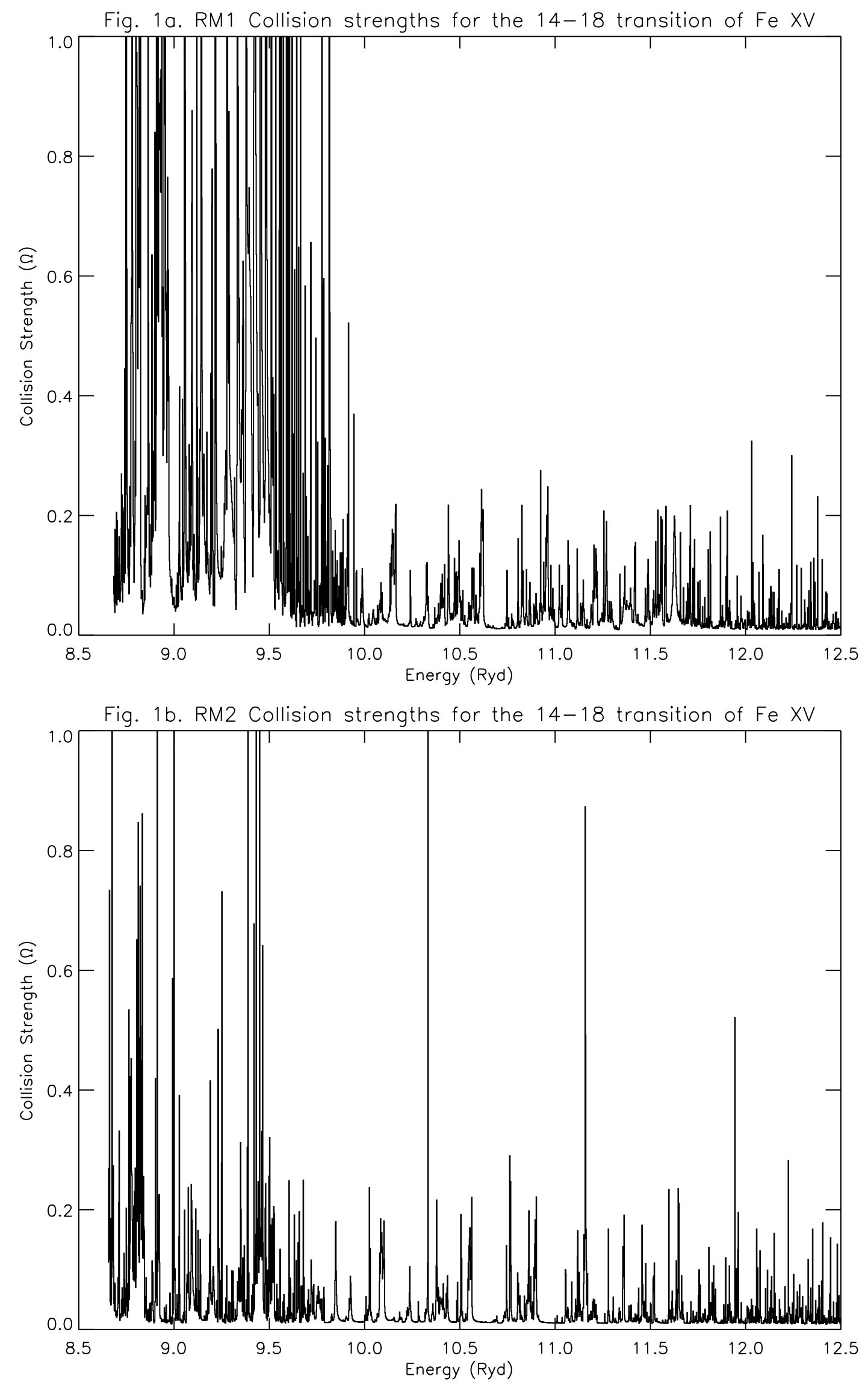

Fig. 1. Collision strengths for the $\left(1 \mathrm{~s}^{2} 2 \mathrm{~s}^{2} 2 \mathrm{p}^{6}\right) 3 \mathrm{~s} 3 \mathrm{~d}^{1} \mathrm{D}_{2}-3 \mathrm{p} 3 \mathrm{~d}^{3} \mathrm{~F}_{4}^{0}(14-18)$ transition of Fe XV. a) Present results, b) results of Eissner et al. (1999). 
Table 5. Comparison of the present (RM1) effective collision strengths with those of Eissner et al. (RM2: 1999) for the 3s3d-3p3d transitions at three temperatures of $\log T_{\mathrm{e}}=5.0,6.0$ and $7.0 \mathrm{~K} .\left(a \pm b \equiv a \times 10^{ \pm b}\right.$.)

\begin{tabular}{|c|c|c|c|c|c|c|c|}
\hline \multicolumn{2}{|c|}{ Transition } & \multicolumn{3}{|c|}{$\overline{\text { RM1 }}$} & \multicolumn{3}{|c|}{$\overline{\text { RM2 }}$} \\
\hline$i$ & $j$ & 5.0 & 6.0 & 7.0 & 5.0 & 6.0 & 7.0 \\
\hline 11 & 15 & $2.143-0$ & $2.216-0$ & $3.216-0$ & $1.570-0$ & $2.080-0$ & $3.260-0$ \\
\hline 11 & 16 & $1.075-1$ & $3.922-2$ & $7.435-3$ & $6.980-2$ & $2.990-2$ & $6.100-3$ \\
\hline 11 & 17 & $3.491-1$ & $2.831-1$ & $3.761-1$ & $2.760-1$ & $2.790-1$ & $4.090-1$ \\
\hline 11 & 18 & $6.185-2$ & $1.402-2$ & $1.843-3$ & $3.260-2$ & $7.330-3$ & $9.340-4$ \\
\hline 11 & 19 & $4.213-1$ & $4.324-1$ & $5.918-1$ & $3.420-1$ & $4.260-1$ & $6.230-1$ \\
\hline 11 & 20 & $2.221-1$ & $2.152-1$ & 2.779-1 & $1.770-1$ & $2.060-1$ & $2.820-1$ \\
\hline 11 & 21 & $2.978-2$ & $1.348-2$ & $2.639-3$ & $2.330-2$ & $1.020-2$ & $2.100-3$ \\
\hline 11 & 22 & $6.174-1$ & $6.762-1$ & $9.593-1$ & $5.020-1$ & $6.610-1$ & $1.010-0$ \\
\hline 11 & 23 & $1.321-0$ & $1.443-0$ & $2.055-0$ & $1.060-0$ & $1.390-0$ & $2.100-0$ \\
\hline 11 & 24 & $4.825-1$ & $5.038-1$ & $6.958-1$ & $3.790-1$ & $4.680-1$ & $6.850-1$ \\
\hline 11 & 25 & $3.938-2$ & $2.453-2$ & $5.145-3$ & $3.570-2$ & $1.580-2$ & $3.590-3$ \\
\hline 11 & 26 & $2.037-2$ & $1.236-2$ & $5.727-3$ & $1.220-2$ & $7.730-3$ & $4.740-3$ \\
\hline 12 & 15 & $8.571-1$ & $6.493-1$ & $8.734-1$ & $5.860-1$ & $5.920-1$ & $8.810-1$ \\
\hline 12 & 16 & $3.337-0$ & $3.583-0$ & $5.213-0$ & $2.350-0$ & $3.380-0$ & $5.330-0$ \\
\hline 12 & 17 & $2.501-1$ & $1.052-1$ & $9.752-2$ & $1.900-1$ & $9.020-2$ & $9.280-2$ \\
\hline 12 & 18 & $1.390-1$ & $4.943-2$ & $9.416-3$ & $9.250-2$ & $3.670-2$ & $7.580-3$ \\
\hline 12 & 19 & $1.355-0$ & $1.460-0$ & $2.088-0$ & $1.060-0$ & $1.400-0$ & $2.130-0$ \\
\hline 12 & 20 & $2.083-1$ & $1.822-1$ & $2.125-1$ & $1.790-1$ & $1.890-1$ & $2.430-1$ \\
\hline 12 & 21 & 7.959-1 & $8.165-1$ & $1.126-0$ & $6.270-1$ & $7.590-1$ & $1.100-0$ \\
\hline 12 & 22 & $1.239-2$ & $8.466-3$ & $2.067-3$ & $8.930-3$ & $5.710-3$ & $1.570-3$ \\
\hline 12 & 23 & 5.199-1 & $5.461-1$ & $7.533-1$ & 4.390-1 & $5.520-1$ & $8.190-1$ \\
\hline 12 & 24 & $2.131-0$ & $2.305-0$ & $3.284-0$ & $1.710-0$ & $2.220-0$ & $3.350-0$ \\
\hline 12 & 25 & $7.950-2$ & $5.700-2$ & 1.974-2 & $7.430-2$ & $3.550-2$ & $1.610-2$ \\
\hline 12 & 26 & $4.422-2$ & $3.165-2$ & $1.965-2$ & $3.050-2$ & $2.170-2$ & $1.740-2$ \\
\hline 13 & 15 & $2.428-1$ & $4.756-2$ & $1.846-2$ & $1.090-1$ & $2.650-2$ & $1.590-2$ \\
\hline 13 & 16 & $1.052-0$ & $8.660-1$ & $1.179-0$ & $6.230-1$ & $7.730-1$ & $1.170-0$ \\
\hline 13 & 17 & $4.498-1$ & $2.223-1$ & $2.335-1$ & $2.970-1$ & $1.810-1$ & $2.160-1$ \\
\hline 13 & 18 & $5.213-0$ & $5.650-0$ & $8.173-0$ & $3.760-0$ & $5.300-0$ & $7.090-0$ \\
\hline 13 & 19 & $3.199-2$ & $1.378-2$ & $2.767-3$ & $2.290-2$ & $1.030-2$ & $2.270-3$ \\
\hline 13 & 20 & $2.621-0$ & $2.835-0$ & $4.055-0$ & $2.060-0$ & $2.740-0$ & $4.190-0$ \\
\hline 13 & 21 & $3.304-0$ & $3.489-0$ & $4.906-0$ & $2.690-0$ & $3.380-0$ & $5.020-0$ \\
\hline 13 & 22 & $1.187-2$ & $6.640-3$ & $9.643-4$ & $9.230-3$ & $5.600-3$ & $8.280-4$ \\
\hline 13 & 23 & $3.609-2$ & $2.072-2$ & $3.502-3$ & $2.660-2$ & $1.610-2$ & $2.790-3$ \\
\hline 13 & 24 & 4.356-1 & $4.247-1$ & $5.476-1$ & $3.850-1$ & $4.510-1$ & $6.360-1$ \\
\hline 13 & 25 & $1.324-1$ & $1.129-1$ & $5.600-2$ & $1.230-1$ & $6.520-2$ & $4.440-2$ \\
\hline 13 & 26 & $4.603-2$ & $2.290-2$ & $4.409-3$ & $3.120-2$ & $1.780-2$ & $3.700-3$ \\
\hline 14 & 15 & $3.888-1$ & $2.351-1$ & $2.814-1$ & $2.070-1$ & $1.970-1$ & $2.920-1$ \\
\hline 14 & 16 & $3.340-1$ & $1.066-1$ & $6.214-2$ & $1.050-1$ & $5.310-2$ & $5.170-2$ \\
\hline 14 & 17 & $1.821-0$ & $1.483-0$ & $2.042-0$ & $1.150-0$ & $1.350-0$ & $2.090-0$ \\
\hline 14 & 18 & $3.563-1$ & $8.960-2$ & $1.260-2$ & $1.000-1$ & $2.980-2$ & $5.560-3$ \\
\hline 14 & 19 & $1.129-1$ & $5.060-2$ & $3.926-2$ & $5.920-2$ & $3.960-2$ & $3.790-2$ \\
\hline 14 & 20 & $2.072-1$ & $9.027-2$ & $6.797-2$ & $1.060-1$ & $6.670-2$ & $5.720-2$ \\
\hline 14 & 21 & $2.871-1$ & $9.954-2$ & $4.675-2$ & $1.550-1$ & $7.740-2$ & $4.310-2$ \\
\hline 14 & 22 & $3.500-2$ & $8.987-3$ & $1.526-3$ & $1.500-2$ & $5.350-3$ & $1.100-3$ \\
\hline 14 & 23 & $1.023-1$ & $3.212-2$ & $6.026-3$ & $5.530-2$ & $2.330-2$ & $4.850-3$ \\
\hline 14 & 24 & $1.843-1$ & $6.267-2$ & $1.525-2$ & $1.150-1$ & $5.270-2$ & $1.570-2$ \\
\hline 14 & 25 & $8.490-0$ & $9.026-0$ & $1.295+1$ & $8.520-0$ & $9.200-0$ & $1.370+1$ \\
\hline 14 & 26 & $2.252-0$ & $2.445-0$ & $3.472-0$ & $2.430-0$ & $2.520-0$ & $3.730-0$ \\
\hline
\end{tabular}

these results. In spite of being aware of this deficiency in our For example, collision rates in Mg-like ions are particularly calculations, we have preferred to report the values of $\Upsilon$ up to susceptible to non-Maxwellian electron distributions. These $T_{\mathrm{e}}=10^{7} \mathrm{~K}$, because data at high temperatures are required. may arise in, for example, the solar transition region and 
Table 6. Comparison of the present (RM1) effective collision strengths with those of Eissner et al. (RM2: 1999) for the $3 \mathrm{~s} 3 \mathrm{~d}-3 \mathrm{~d}^{2}$ transitions at three temperatures of $\log T_{\mathrm{e}}=5.0,6.0$ and $7.0 \mathrm{~K} .\left(a \pm b \equiv a \times 10^{ \pm b}.\right)$

\begin{tabular}{|c|c|c|c|c|c|c|c|}
\hline \multicolumn{2}{|c|}{ Transition } & \multicolumn{3}{|c|}{$\overline{\text { RM1 }}$} & \multicolumn{3}{|c|}{$\overline{\text { RM2 }}$} \\
\hline$i$ & $j$ & 5.0 & 6.0 & 7.0 & 5.0 & 6.0 & 7.0 \\
\hline 11 & 27 & $1.648-1$ & $1.716-1$ & $1.930-1$ & $1.620-1$ & $1.730-1$ & $1.960-1$ \\
\hline 11 & 28 & $9.135-2$ & $9.220-2$ & $9.940-2$ & $8.990-2$ & $9.280-2$ & $1.010-1$ \\
\hline 11 & 29 & $7.485-3$ & $4.652-3$ & $1.705-3$ & $6.560-3$ & $4.420-3$ & $1.670-3$ \\
\hline 11 & 30 & $2.823-2$ & $2.074-2$ & $1.487-2$ & $2.730-2$ & $2.570-2$ & $2.150-2$ \\
\hline 11 & 31 & $3.365-3$ & $2.640-3$ & $1.018-3$ & $3.250-3$ & $2.590-3$ & $1.010-3$ \\
\hline 11 & 32 & $7.631-2$ & $8.069-2$ & $9.235-2$ & $7.590-2$ & $8.180-2$ & $9.430-2$ \\
\hline 11 & 33 & $1.988-2$ & $1.948-2$ & $2.158-2$ & $1.230-2$ & $1.310-2$ & $1.500-2$ \\
\hline 11 & 34 & $1.912-2$ & $1.457-2$ & $5.546-3$ & $1.730-2$ & $1.410-2$ & $5.450-3$ \\
\hline 11 & 35 & $2.187-3$ & $1.555-3$ & $5.382-4$ & $1.570-3$ & $1.250-3$ & $4.790-4$ \\
\hline 12 & 27 & $1.292-1$ & $1.306-1$ & $1.413-1$ & $1.270-1$ & $1.320-1$ & $1.440-1$ \\
\hline 12 & 28 & $2.069-1$ & $2.148-1$ & $2.418-1$ & $2.040-1$ & $2.170-1$ & $2.460-1$ \\
\hline 12 & 29 & $1.037-1$ & $1.021-1$ & $1.390-1$ & $1.010-1$ & $1.020-1$ & $1.090-1$ \\
\hline 12 & 30 & $5.054-2$ & $3.599-2$ & $2.723-2$ & $5.260-2$ & $5.230-2$ & $5.080-2$ \\
\hline 12 & 31 & $5.508-2$ & $5.856-2$ & $6.777-2$ & $5.480-2$ & $5.940-2$ & $6.930-2$ \\
\hline 12 & 32 & $2.436-2$ & $2.296-2$ & $2.017-2$ & $2.370-2$ & $2.300-2$ & $2.050-2$ \\
\hline 12 & 33 & $8.822-2$ & $8.884-2$ & $9.826-2$ & $6.610-2$ & $6.970-2$ & $7.720-2$ \\
\hline 12 & 34 & $3.312-2$ & $2.496-2$ & $9.478-3$ & $2.950-2$ & $2.400-2$ & $9.280-3$ \\
\hline 12 & 35 & $4.105-3$ & $2.798-3$ & $9.812-4$ & $2.810-3$ & $2.250-3$ & $8.810-4$ \\
\hline 13 & 27 & $2.023-2$ & $1.704-2$ & $1.536-2$ & $1.920-2$ & $1.690-2$ & $1.550-2$ \\
\hline 13 & 28 & $1.405-1$ & $1.398-1$ & $1.491-1$ & $1.390-1$ & $1.410-1$ & $1.520-1$ \\
\hline 13 & 29 & $4.518-1$ & $4.664-1$ & $5.307-1$ & $4.440-1$ & $4.700-1$ & $5.290-1$ \\
\hline 13 & 30 & $8.070-2$ & $5.375-2$ & $4.627-2$ & $7.510-2$ & $7.860-2$ & $8.550-2$ \\
\hline 13 & 31 & $3.282-3$ & $2.471-3$ & $9.436-4$ & $3.020-3$ & $2.380-3$ & $9.210-4$ \\
\hline 13 & 32 & $8.416-2$ & $8.734-2$ & $9.691-2$ & $8.310-2$ & $8.820-2$ & $9.900-2$ \\
\hline 13 & 33 & $1.549-1$ & $1.502-1$ & $1.557-1$ & $1.180-1$ & $1.190-1$ & $1.210-1$ \\
\hline 13 & 34 & $4.963-2$ & $3.672-2$ & $1.424-2$ & $4.310-2$ & $3.510-2$ & $1.390-2$ \\
\hline 13 & 35 & $5.735-3$ & $3.976-3$ & $1.418-3$ & $4.270-3$ & $3.400-3$ & $1.300-3$ \\
\hline 14 & 27 & $2.249-2$ & $1.487-2$ & $5.900-3$ & $2.150-2$ & $1.470-2$ & $5.910-3$ \\
\hline 14 & 28 & $3.094-2$ & $2.038-2$ & $7.609-3$ & $3.090-2$ & $2.030-2$ & $7.600-3$ \\
\hline 14 & 29 & $4.084-2$ & $2.692-2$ & $1.058-2$ & $4.260-2$ & $2.710-2$ & $1.020-2$ \\
\hline 14 & 30 & $2.729-1$ & $2.528-1$ & $2.753-1$ & $1.730-1$ & $1.840-1$ & $2.100-1$ \\
\hline 14 & 31 & $3.192-3$ & $2.240-3$ & $8.314-4$ & $2.790-3$ & $2.100-3$ & $8.030-4$ \\
\hline 14 & 32 & $9.374-3$ & $6.679-3$ & $2.454-3$ & $8.300-3$ & $6.320-3$ & $2.370-3$ \\
\hline 14 & 33 & $8.776-2$ & $7.976-2$ & $8.116-2$ & $1.300-1$ & $1.360-1$ & $1.530-1$ \\
\hline 14 & 34 & $3.662-1$ & $3.783-1$ & $4.189-1$ & $3.830-1$ & $4.140-1$ & $4.640-1$ \\
\hline 14 & 35 & $5.419-2$ & $5.642-2$ & $6.371-2$ & $5.270-2$ & $5.510-2$ & $6.330-2$ \\
\hline
\end{tabular}

provide a "high energy tail" to the electron distribution with a corresponding increase in the collision rate, as noted by Keenan et al. (1989) and Pinfield et al. (1999).

Although the presently reported results for $\Upsilon$ are the most reliable available today, yet there is scope for improvement. These improvements can be made particularly in three areas, which are: wavefunctions, partial wave range, and energy range. Our present wavefunctions are very good and should be better than those of other workers. This can be judged from the agreement between the theoretical and experimental energy levels. The other indicator is the agreement between the length and velocity forms of the oscillator strengths. For a large calculation such as the present one, it is difficult to get a reasonably good agreement between the two forms for all the allowed transitions, yet there is scope for improvement. We tried to resolve this problem in our earlier work (Deb et al. 1999) but without any definitive conclusion. The range of partial waves included in the present work is fully sufficient for the convergence of $\Omega$ at lower energies, but at other energies a higher range is preferable. Although to take account of the higher missing partial waves top-up has been included, a higher range will provide more definitive values of $\Omega$. Similarly, as noted above, extension of the energy range of $\Omega$ calculations will improve the results of $\Upsilon$ at higher temperatures. Our currently available computational resources do not allow us to incorporate these improvements, but we hope that it may be possible in future. Until then, we expect the present results will be highly useful for plasma diagnostics. 
Acknowledgements. This work has been financed by the Engineering and Physical Sciences Research Council of the United Kingdom, the National Science Foundation and Division of Chemical Sciences, Office of Basic Energy Sciences, DoE of the United States, and NATO grant CRG 930722. We wish to thank Dr. Patrick Norrington for discussions and for making his code available to us prior to publication. Also, KMA would like to thank Dr. Claudio Mendoza for discussions and for providing the collision strength data in numerical form, which made possible the comparisons shown in the paper.

\section{References}

Acton, L. W., Bruner, M. E., Brown, W. A., et al. 1985, ApJ, 291, 865 Aggarwal, K. M. 1998, ApJS, 118, 589

Aggarwal, K. M., Deb, N. C., Keenan, F. P., \& Msezane, A. Z. 1999, J. Phys. B, 32, 5257

Aggarwal, K. M., Deb, N. C., Keenan, F. P., \& Msezane, A. Z. 2000, J. Phys. B, 33, L391

Aggarwal, K. M., Keenan, F. P., \& Msezane, A. Z. 2001, J. Phys. B, 34, L757

Burgess, A., \& Tully, J. A. 1978, J. Phys. B, 24, 4271

Churilov, S. S., Kononov, E. Y., Ryabtsev, A. N., \& Zayikin, Y. F. 1985, Phys. Scr., 32, 501
Churilov, S. S., Levashov, V. E., \& Wyart, J. F. 1989, Phys. Scr., 40, 625

Deb, N. C., Aggarwal, K. M., \& Msezane, A. Z. 1999, ApJS, 121, 265

Dyall, K. G., Grant, I. P., Johnson, C. T., Parpia, F. A., \& Plummer, E. P. 1989, Comput. Phys. Commun., 55, 424

Eissner, W., Galavis, M. E., Mendoza, C., \& Zeippen, C. J. 1999, ApJS, 137, 165

Eissner, W., Jones., M., \& Nussbaumer, H. 1974, Comput. Phys. Commun., 8, 271

Froese-Fischer, C. 1991, Comput. Phys. Commun., 64, 369

Griffin, D. C., Badnell, N. R., Pindzola, M. S., \& Shaw, J. A. 1999, J. Phys. B, 32, 2139+4129

Hibbert, A. 1975, Comput. Phys. Commun. 9, 141

Keenan, F. P., Cook, J. W., Dufton, P. L., \& Kingston, A. E. 1989, ApJ, 340,1135

Litzen, U., \& Redfors, A. 1987, Phys. Scr., 36, 895

Norrington, P. H., \& Grant, I. P. 2003, Comput. Phys. Commun., in preparation

Pinfield, D. J., Keenan, F. P., Mathioudakis, M., et al. 1999, ApJ, 527, 1000

Sugar, J., \& Corliss, C. 1985, J. Phys. Chem. Ref. Data Suppl., 2, 14, 484 